\title{
Feshbach molecules in a one-dimensional Fermi gas
}

\author{
D. B. M. Dickerscheid* and H. T. C. Stoof \\ Institute for Theoretical Physics, University of Utrecht, Princetonplein 5, 3584 CC Utrecht, The Netherlands
}

(Received 21 June 2005; published 21 November 2005)

\begin{abstract}
We consider the binding energy and the wave function of Feshbach molecules confined in a one-dimensional matter waveguide. We compare the binding energy with the experiment of Moritz et al. [Phys. Rev. Lett. 94, 210401 (2005)] and find excellent agreement for the full magnetic field range explored experimentally.
\end{abstract}

DOI: 10.1103/PhysRevA.72.053625

PACS number(s): 03.75.Ss, 05.30.Fk, 34.50.-s, 71.10.Pm

\section{INTRODUCTION}

In a beautiful experiment, Moritz et al. recently reported the observation of two-particle bound states of ${ }^{40} \mathrm{~K}$ confined in a one-dimensional matter waveguide [1]. In the experiment, an array of equivalent one-dimensional (1D) quantum system is realized by trapping a mixture of two hyperfine states of ${ }^{40} \mathrm{~K}$ atoms in a two-dimensional optical lattice. The atoms are trapped at the intensity maxima and the radial confinement is only a fraction of the lattice period. At a given value of the magnetic field, the binding energy $E_{B}$ of the bound states is probed by radio-frequency spectroscopy.

Although Moritz et al. noted its limitations, the description of the experiment mentions the use of a single-channel model of radially confined atoms interacting with a pseudopotential [2,3]. Within this model, the bound-state energy $E_{B}$ is related to the $s$-wave scattering length $a$ of the atoms by

$$
\frac{a}{a_{\perp}}=-\frac{\sqrt{2}}{\zeta\left(1 / 2,1 / 2-E_{B} / 2 \hbar \omega_{\perp}\right)},
$$

where $a_{\perp}=\sqrt{\hbar / m \omega_{\perp}}, m$ is the atomic mass, and $\omega_{\perp}$ is the radial trapping frequency. To vary the scattering length however the experiment makes use of a Feshbach resonance at a magnetic field of $B_{0}=202.1 \mathrm{G}$. For such a Feshbach resonance, a two-channel approach is physically more realistic.

For the Feshbach problem, the molecular binding energy $E_{B}$ always satisfies the equation [4],

$$
E_{B}-\delta(B)=\hbar \Sigma\left(E_{B}\right) .
$$

Here the detuning $\delta(B)=\Delta \mu\left(B-B_{0}\right)$ varies as a function of the magnetic field and depends on the difference in magnetic moments $\Delta \mu$ between the open and closed channels in the Feshbach problem. The resonance is located at the magnetic field strength $B_{0}$. For the homogeneous Fermi gas the molecular self-energy is given by [4]

$$
\hbar \Sigma(E)=-\left(\frac{g^{2} m^{3 / 2}}{4 \pi \hbar^{3}}\right) \frac{i \sqrt{E}}{1-i\left|a_{\mathrm{bg}}\right| \sqrt{m E / \hbar^{2}}},
$$

which leads to corrections to the single-channel result $-\hbar^{2} / m a^{2}$. Here $g=\hbar \sqrt{4 \pi a_{\mathrm{bg}} \Delta B \Delta \mu / m}$ is the atom-molecule coupling, $\Delta B$ is the width of the Feshbach resonance, $\Delta \mu$ is the difference in magnetic moments, and $a_{\mathrm{bg}}$ is the back-

\footnotetext{
*Electronic address: dickrsch@phys.uu.nl
}

ground scattering length. In Fig. 1 we show for this threedimensional case the molecular binding energy for both the single and two-channel approaches, respectively. Whereas the single-channel results deviate significantly from the experimental data, there is an excellent agreement with the two-channel theory. It is therefore a priori not clear that in the one-dimensional case the single-channel theory as given by Eq. (1) is sufficiently accurate for the full range of magnetic fields explored by the experiment. In the following we derive the self-energy for the confined case and make a comparisson with the experimental data.

\section{THEORY}

Two atoms in a waveguide near a Feshbach resonance are described by the following Hamiltonian

$$
H=H_{\mathrm{a}}+H_{\mathrm{m}}+V_{\mathrm{am}} .
$$

Here $H_{\mathrm{a}}$ represents the atomic contribution, $H_{\mathrm{m}}$ describes the bare molecules, and $V_{\text {am }}$ is the atom-molecule coupling. Explicitly we have for the atoms

$$
H_{\mathrm{a}}=\sum_{i=1,2}\left\{K_{i}+\frac{m \omega_{\perp}^{2}}{2}\left(x_{i}^{2}+y_{i}^{2}\right)\right\}+V_{\mathrm{aa}} \delta(\mathbf{r}),
$$

with $K_{i}=-\hbar^{2} \nabla_{i}^{2} / 2 m$ the kinetic energy of atom $i, V_{\text {aa }}$ the strength of the nonresonant atom-atom interaction, and $\mathbf{r}$ the

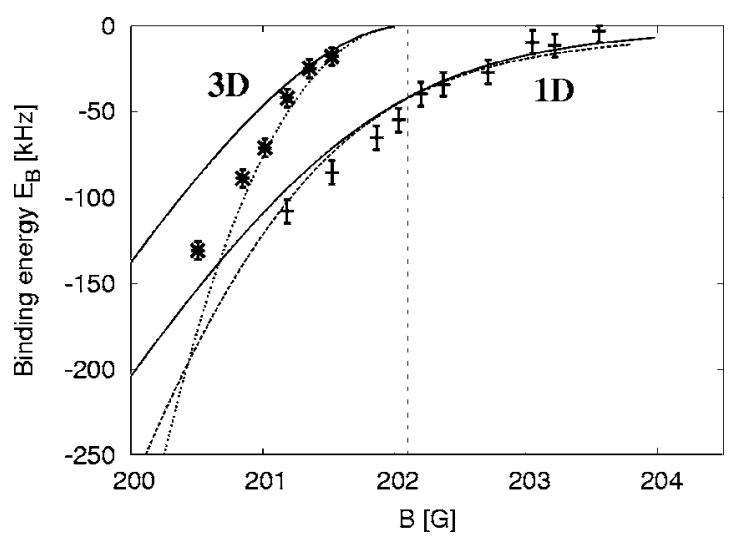

FIG. 1. Binding energies for 1D and 3D molecules as a function of the magnetic field. The solid lines correspond to the singlechannel result. The dashed lines are calculated within the twochannel theory. 


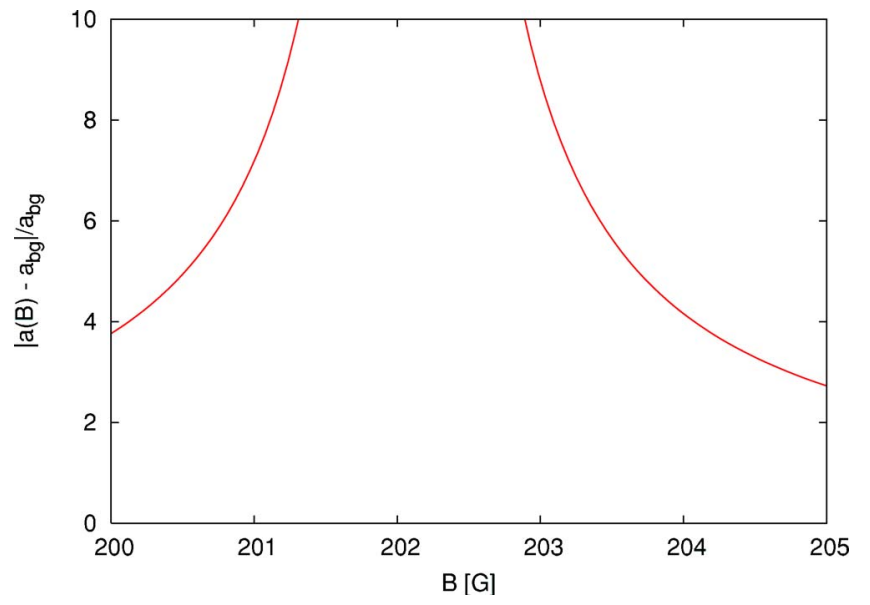

FIG. 2. (Color online) The ratio of the resonant part of the $s$-wave scattering length and the background scattering length $a_{\mathrm{bg}}$.

relative coordinate of the two atoms. The atoms are coupled to a molecular channel with a coupling $V_{\mathrm{am}}$. We show in Fig. 2 that near the resonance we have that $V_{\text {aa }} \ll V_{\text {am }}$, which allows us to neglect the nonresonant atom-atom interaction in that case. Note that outside the magnetic field range considered in Figs. 1 and 2 there are, in principle, corrections to the molecular binding energy coming from the background atom-atom scattering.

For two atoms in the waveguide, the two-channel Feshbach problem in the relative coordinate, after splitting off the center-of-mass motion, is then given by

$$
\left(\begin{array}{cc}
H_{0} & V_{\mathrm{am}} \\
V_{\mathrm{am}} & \delta_{B}
\end{array}\right)\left(\begin{array}{c}
\left|\psi_{\mathrm{a}}\right\rangle \\
\left|\psi_{\mathrm{m}}\right\rangle
\end{array}\right)=E\left(\begin{array}{c}
\left|\psi_{\mathrm{a}}\right\rangle \\
\left|\psi_{\mathrm{m}}\right\rangle
\end{array}\right)
$$

Here the atomic Hamiltonian is $H_{0}=-\hbar^{2} \nabla_{\mathbf{r}}^{2} / m+m \omega_{\perp}^{2} \mathbf{r}_{\perp}^{2} / 4$, where $\nabla_{\mathbf{r}}^{2}=\partial_{\perp}^{2}+\partial_{z}^{2}$, and $\mathbf{r}_{\perp}$ is the radial component of $\mathbf{r}$. Only the relative part is relevant here, since only this part contains the interaction between the atoms. The bare detuning is denoted by $\delta_{B}$. The eigenstates $\left|\psi_{n, k_{z}}\right\rangle$ of $H_{0}$ that are relevant for an $s$-wave Feshbach resonance are a product state of a twodimensional harmonic oscillator wave function in the radial direction and a plane wave along the axial direction. The associated energies are given by $E_{n, k_{z}}=(2 n+1) \hbar \omega_{\perp}$ $+\hbar^{2} k_{z}^{2} / m$. The eigenstates of the two-dimensional harmonic oscillator that are relevant for $s$-wave scattering can be written as $\psi_{n}\left(r_{\perp}, \phi\right)=\left(2 \pi a_{\perp}^{2}\right)^{-1 / 2} e^{-r_{\perp}^{2} / 4 a_{\perp}^{2}} L_{n}^{(0)}\left(r_{\perp}^{2} / 2 a_{\perp}^{2}\right)$, where $L_{n}^{(0)}(x)$ is the generalized Laguerre polynomial and $\hbar \omega_{\perp}$ $=\hbar^{2} / m a_{\perp}^{2}$. From Eq. (6) we obtain the following equation determining the binding energy of the molecules:

$$
\left\langle\psi_{\mathrm{m}}\left|V_{\mathrm{am}} \frac{1}{E-H_{0}} V_{\mathrm{am}}\right| \psi_{\mathrm{m}}\right\rangle=E-\delta_{B}
$$

Using the above-mentioned eigenstates of $H_{0}$, Eq. (7) can be written as

$$
\sum_{n=0}^{\infty} \int \frac{d k_{z}}{2 \pi} \frac{\left|\left\langle\psi_{\mathrm{m}}\left|V_{\mathrm{am}}\right| \psi_{n, k_{z}}\right\rangle\right|^{2}}{E-E_{n, k_{z}}}=E-\delta_{B}
$$

Using the usual pseudopotential approximation for the atom-molecule coupling, we have $\left\langle\mathbf{r}\left|V_{\mathrm{am}}\right| \psi_{\mathrm{m}}\right\rangle=g \delta(\mathbf{r})$. Substituting this and performing the $k_{z}$ integration we obtain

$$
E-\delta_{B}=\lim _{r_{\perp} \downarrow 0} \frac{-g^{2} m}{\sqrt{2}\left(4 \pi a_{\perp} \hbar^{2}\right)} \times \sum_{n=0}^{\infty} \frac{e^{-r_{\perp}^{2} / 4 a_{\perp}^{2}} L_{n}^{(0)}\left(r_{\perp}^{2} / 2 a_{\perp}^{2}\right)}{\sqrt{n+1 / 2-E / 2 \hbar \omega_{\perp}}} .
$$

The inverse square root $1 / \sqrt{n+1 / 2-E / 2 \hbar \omega_{\perp}}$ in the summand can be represented by the gaussian integral $(2 / \sqrt{\pi}) \int_{0}^{\infty} d t e^{-\left(n+1 / 2-E / 2 \hbar \omega_{\perp}\right) t^{2}}$. To evaluate the sum over $n$ we substitute the above integral representation. The dependence on $n$ of the summand appears now in the exponent and in the degree of the Laguerre polynomial. As a result, the sum can be directly evaluated by making use of the generating functions of the Laguerre polynomials,

$$
\sum_{n=0}^{\infty} L_{n}^{(0)}(x) z^{n}=(1-z)^{-1} \exp \left(\frac{x z}{z-1}\right) \text {. }
$$

In our case we have $z=e^{-t^{2}}$. Using this result and making the transformation $y=t^{2}$ we arrive at

$$
\begin{aligned}
E-\delta_{B}= & \lim _{r_{\perp} \downarrow 0} \frac{-g^{2} m}{\sqrt{2 \pi}\left(4 \pi a_{\perp} \hbar^{2}\right)} \int_{0}^{\infty} \exp \left(\frac{r_{\perp}^{2}}{2 a_{\perp}^{2}} \frac{e^{-y}}{e^{-y}-1}\right) \\
& \times \frac{\exp \left\{-\left(1 / 2-E / 2 \hbar \omega_{\perp}\right) y\right\}}{\sqrt{y}\left(1-e^{-y}\right)} d y .
\end{aligned}
$$

For small values of $y$ the integrand in the above equation behaves as $y^{-3 / 2} e^{-r^{2} / 2 y}$. Note that we have

$$
\frac{1}{\sqrt{\pi}} \int_{0}^{\infty} d y y^{-3 / 2} e^{-r^{2} / 2 y}=\sqrt{2} / r
$$

We add and subtract this integral from Eq. (11) and in doing so we explicitly split off the $1 / r$ divergence from the sum. The divergence in the self-energy is energy independent and is related to the ultraviolet divergence that comes about because we have used pseudopotentials. To deal with this divergence, we have to use the renormalized detuning instead of the bare detuning. The former is defined as $\delta=\delta_{B}$ $-\lim _{r \downarrow 0} m g^{2} / 4 \pi \hbar^{2} r$, where $\delta=\Delta \mu\left(B-B_{0}\right)$ is determined by the experimental value of the magnetic field $B_{0}$ at resonance and the magnetic moment difference $\Delta \mu=16 / 9$ Bohr magneton for the ${ }^{40} \mathrm{~K}$ atoms of interest. Note that, as expected, the required subtraction is exactly equal to the one needed in the absence of the optical lattice. In the latter case we have to subtract $g^{2} \int d \mathbf{k} m / \hbar^{2} \mathbf{k}^{2}(2 \pi)^{3}$ [4-6], which can be interpreted as $\delta=\delta_{\mathrm{B}}-\lim _{\mathbf{r} \downarrow 0} g^{2} \int d \mathbf{k} e^{i \mathbf{k} \cdot \mathbf{r}} m / \hbar^{2} \mathbf{k}^{2}(2 \pi)^{3}$. Using the renormalized detuning, we find that the binding energy of the dressed molecules satisfies the desired equation

$$
E_{B}-\delta(B)=\hbar \Sigma\left(E_{B}\right)
$$

where the molecular self-energy for the harmonically confined one-dimensional system is given by 


$$
\hbar \Sigma(E)=-\frac{m g^{2}}{\sqrt{2}\left(4 \pi a_{\perp} \hbar^{2}\right)} \zeta\left(1 / 2,1 / 2-E / 2 \hbar \omega_{\perp}\right) .
$$

\section{RESULTS AND DISCUSSION}

Using the self-energy for the confined gas we can now solve for the binding energy in Eq. (13). The result is also shown in Fig. 1. We find an improved description of the experiment, although the differences with the single-channel prediction are small near resonance and only become large for larger detunings. This presents one way in which to experimentally probe these differences. Alternatively, it is also possible to directly measure the bare molecule fraction $Z$ of the dressed molecules [7], which is always equal to zero in the single-channel model. To be concrete, we have for the dressed molecular wave function

$$
\left|\psi_{\text {dressed }}\right\rangle=\sqrt{Z}\left|\psi_{\text {closed }}\right\rangle+\sqrt{1-Z}\left|\psi_{\text {open }}\right\rangle,
$$

where $\left|\psi_{\text {closed }}\right\rangle$ is the wave function of the bare molecules and $\left|\psi_{\text {open }}\right\rangle$ denotes the wave function of the atom pair in the open channel of the Feshbach resonance. With this application in mind, we have plotted in Fig. 3 the probability

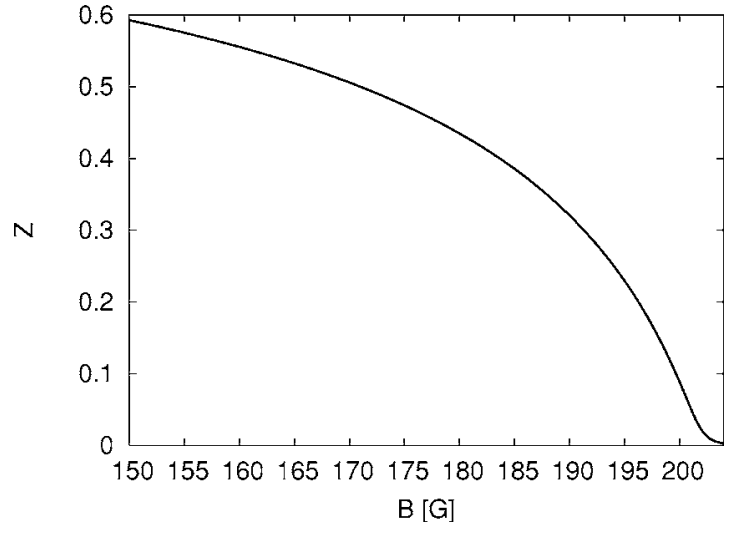

FIG. 3. The bare molecule fraction $Z$ as a function of the magnetic field.

$Z$, which is determined from the above self-energy by $Z=1 /\left(1-\partial \hbar \Sigma\left(E_{B}\right) / \partial E_{B}\right)$.

This work was supported by the Stichting voor Fundamenteel Onderzoek der Materie (FOM) and by the Nederlandse Organisatie voor Wetenschaplijk Onderzoek (NWO).
[1] H. Moritz, T. Stöferle, K. Güenter, M. Köhl, and T. Esslinger, Phys. Rev. Lett. 94, 210401 (2005).

[2] T. Bergeman, M. G. Moore, and M. Olshanii, Phys. Rev. Lett. 91, 163201 (2003).

[3] T. Busch, B.-G. Englert, K. Rzazewski, and M. Wilkens, Found. Phys. 28, 549 (1998).

[4] R. A. Duine and H. T. C. Stoof, Phys. Rep. 396, 115 (2004).
[5] D. B. M. Dickerscheid, U. Al Khawaja, D. van Oosten, and H. T. C. Stoof, Phys. Rev. A 71, 043604 (2005).

[6] S. J. J. M. F. Kokkelmans and M. J. Holland, Phys. Rev. Lett. 89, 180401 (2002).

[7] G. B. Partridge, K. E. Strecker, R. I. Kamar, M. W. Jack, and R. G. Hulet, Phys. Rev. Lett. 95, 020404 (2005). 